\title{
Optimal timing for social distancing during an epidemic
}

\author{
Oscar Patterson-Lomba \\ Analysis Group Inc., Boston, MA, USA
}

\begin{abstract}
Social distancing is an effective way to contain the spread of a contagious disease, particularly when facing a novel pathogen and no pharmacological interventions are available. In such cases, conventional wisdom suggests that social distancing measures should be introduced as soon as possible after the beginning of an outbreak to more effectively mitigate the spread of the disease. Using a simple epidemiological model we show that, however, there is in fact an optimal time to initiate a temporal social distancing intervention if the goal is to reduce the final epidemic size or "flatten" the epidemic curve. The optimal timing depends strongly on the effective reproduction number $\left(R_{0}\right)$ of the disease, such that as the $R_{0}$ increases, the optimal time decreases non-linearly. Additionally, if pharmacological interventions (e.g., a vaccine) become available at some point during the epidemic, the sooner these interventions become available the sooner social distancing should be initiated to maximize its effectiveness. Although based on a simple model, we hope that these insights inspire further investigations within the context of more complex and data-driven epidemiological models, and can ultimately help decision makers to improve temporal social distancing policies to mitigate the spread of epidemics.
\end{abstract}

\section{Introduction}

As the SARS-CoV-2 virus sweeps through the globe, the concept of "social distancing" has become mainstream across news outlets $[14,22,21,18]$, government announcements $[2,1]$ and research publications $[16,7,25,26,5,12,4]$. Social distancing is a very effective way to slow down the swift advance of the COVID-19 pandemic, especially given the current lack of effective pharmaceutical interventions, and our limited ability to identify, let alone track, infectious individuals, particularly asymptomatic ones.

Aiming to reduce instances of person to person transmission, social distancing is a measure that is customarily put into action to decrease the number of contacts among individuals [24]. Examples of social distancing include closing of schools, non-essential workplace closures, and avoiding large gatherings (i.e. public transport, concerts, religious gatherings) in which a large number of individuals are in close contact and facilitating the contagion process [15]. In most cases, due to socio-economic and logistic reasons [13], social distancing measures can only be in effect for a finite period of time (e.g., 2 weeks, 1 month, 2 months) that is typically much shorter than the full duration of the epidemic.

Conventional wisdom suggests that the sooner social distancing measures are imple- 
mented, the higher the chances of curtailing the spread of the virus. Prompt and strict social distancing measures can help "flatten the epidemic" (another fashionable meme at the moment), which in turn will help prevent overwhelming the health system, while also buying us time until effective antivirals and vaccines can be deployed on a mass scale [19]. Implementing swift social distancing measures is one of the most effective ways to stop the spread of the virus, and when the facing a novel and very transmissible virus like COVID-19, social distancing is our first and most effective line of defense. However, is it true that the sooner a temporal social distancing measured is introduced the better health outcomes we will get? The answer to this question would be a resounding "yes" if such measures were to last for extended periods of time (i.e., until the epidemic is over or almost over). However, social distancing interventions are seldom extended long enough to drive the epidemic to extinction on its own. In fact, it is known that new epidemic waves can arise after the social distancing measures are lifted if the pool of susceptibles is large enough, as current models suggest $[4,15,11]$, and as it has historically happened in several occasions, including during the 1918 Spanish influenza pandemic [6], the 2003 SARS epidemic in Canada [8], and the 2009 H1N1 influenza epidemic in Mexico [10].

We argue that how soon temporal social distancing should start depends on what are the health outcomes we are optimizing for, and on when other effective interventions (such as antivirals or vaccines) will be available following the social distancing period. If the goal is to reduce the final number of infected cases, or reduce or delay the peak of the epidemic (i.e., flatten the epidemic curve), we show the existence of an optimal timing to initiate the social distancing period.

The goal of this paper is to study how to efficiently time temporal social distancing measures, and investigate how the optimal time to implement a social distancing intervention depends on i) disease transmissibility, ii) the length of the social distancing, iii) its effectiveness in reducing transmissibility, and iv) when will other (pharmacological) interventions will be available. We define "optimal" based on three different epidemiologically relevant criteria: optimal timing of social distancing 1) to minimize the final size of the epidemic; 2) to maximize the delay in the peak of the infection incidence curve of the epidemic; and 3) to minimize the peak of the infection incidence curve of the epidemic. The first criteria aims to minimize the impact of the epidemic in terms of overall infections, while the other two refer to the flattening of the epidemic as to not overburden the health system in place to care for infected individuals.

\section{Methods}

\section{The SIR model with social distancing}

The idea of an optimal social distancing timing is investigated in the context of a classic SIR type model [3]. Individuals are classified based on their infectious status as susceptible $(S)$, infected $(I)$ and recovered $(R)$. The force of infection at time $t$ is given by $\beta(t) I(t)$, where $\beta(t)$ is the transmission probability. This term is the product of the number of contacts 
per capita per time unit, $c(t)$, and the probability of transmission given a contact, $p$; that is $\beta(t)=c(t) p$. The social distancing measure affects the $c(t)$ parameter, such that when social distancing is in effect $c(t)$ is reduced. The corresponding reduction in $\beta(t)$ due to a temporary social distancing measure can be modeled as a "rectangular well" function, as shown in Figure (1). From the start of the epidemic until time $t_{0}$ (the time at which social distancing is initiated), the transmission rate is $\beta$. From time $t_{0}$ to $t_{0}+T$, a social distancing measured is implemented such that the transmission rate is reduced to $\beta_{r}=r \beta$, with $0 \leq r \leq 1$, and $T$ being the length of the period when social distancing measures are in place. Then, after time $t_{0}+T$ (when social distancing ends), the transmission rate comes back up to $\beta$, until time $t_{I}$, at which point a fully effective intervention (e.g., vaccine) is applied such that transmission is completed halted; that is, the effective reproduction number is zero for $t>t_{I}$ (for simplicity we model such scenario by assuming that $\beta=0$ ).

$\beta(t)= \begin{cases}\beta_{r}=r \beta & \text { for } 0 \leq t_{0} \leq t \leq t_{0}+T \\ 0 & \text { for } t_{I} \leq t \\ \beta & \text { otherwise }\end{cases}$

with $0 \leq r \leq 1$

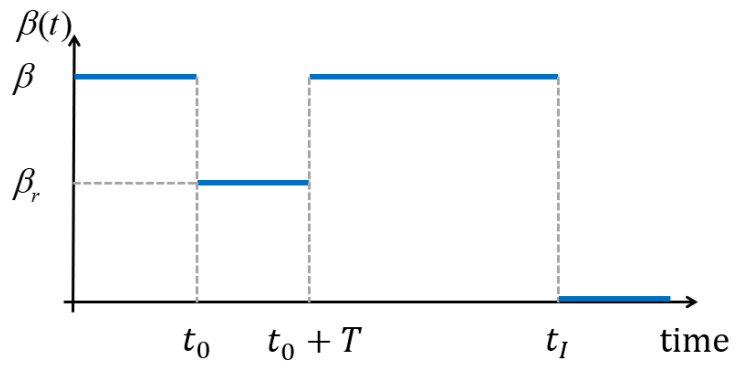

Figure 1: Time-dependent transmission rate. From time $t_{0}$ to $t_{0}+T$, a social distancing measured is implemented such that the transmission rate is reduced by a factor $r$. At time $t_{I}$ an intervention (e.g., vaccine) is applied such that $\beta=0$.

The system of nonlinear differential equations describing the disease spread dynamics is given by

$$
\begin{aligned}
\frac{d S}{d t} & =-\beta(t) S I \\
\frac{d I}{d t} & =\beta(t) S I-\gamma I \\
R(t) & =1-S(t)-I(t)
\end{aligned}
$$

where, with no loss of generality, the size of the population is assumed to be 1 and to remain constant (assuming no births and death processes). The parameter $\gamma$ is the recovery rate. The ODE system in (2)-(3) has a basic reproductive number given by $R_{0}=\beta(t) / \gamma$.

In Appendix 1 we derived the expression for the final epidemic size (i.e., the fraction of infected individuals at the end of the epidemic) in the context of temporal social distancing measure. The resulting expression implicitly relates the final epidemic size, $y$, and $t_{0}$ in a transcendental equation. Thus, $y$ cannot be isolated as to allow a close form solution to the optimal $t_{0}$ that minimizes $y$. 


\section{Numerical simulations setup}

We use numerical simulations of the model in (2)-(3) with the goal to find the optimal $t_{0}$ for reducing the final epidemic size as a function of $R_{0}$, the length of the social distancing intervention $(T)$ and its effectiveness in reducing disease transmissibility $(r)$. The model was simulated for 5 years over a wide range of values for $R_{0}, T$ and $r$ (see Appendix 2 for details). For the first set of simulations, we assumed that $t_{I}=\infty$ (i.e., no pharmacological intervention becomes available during the epidemic).

For each combination of $R_{0}, T$ and $r$, the value of $t_{0}$ corresponding to the minimal final size was obtained. Moreover, to quantify the effect of the optimal social distancing timing, the reduction in final size corresponding to optimal timing (as compared to a scenario with no social distancing) was also computed.

We also use numerical simulations to find the optimal $t_{0}$ for, first, delaying the peak of the epidemic curve, and second, for reducing the peak (i.e., the maximum prevalence of cases), as a function of $R_{0}, T$ and $r$. To quantify the effect of these optimal social distancing timings, the delay in the peak and the reduction in the prevalence peak corresponding to optimal timing (as compared to a scenario with no social distancing) were also computed.

For the next set of simulations we relax the assumption about $t_{I}$, and instead assume that $t_{I} \in(60-730)$ days. For each value of $t_{I}$, it is assumed that $t_{0}+T<t_{I}$. For these simulations, we set $r=0.55$ and $T=30$ days for simplicity. The optimal timing for social distancing is then computed for each value of $R_{0}$ and $t_{I}$.

\section{Results}

\section{Preliminary insights}

Figure (2) show simulations of the model in (2)-(3) with different temporal social distancing scenarios. In particular, we explore the impact of different times at which the social distancing measures are implemented $\left(t_{0}\right)$ in the disease dynamics and the final epidemic size. From Figure (2) (left) we see that if the social distancing is implemented too soon $\left(t_{0}=50\right.$ days), then at the end of the social distancing measure (after 30 days) there are still some infected in the population, and the pool of susceptible is large enough (low population-level immunity) for the disease to take off again and still infect a large portion of the population. Conversely, if social distancing is introduced too late ( $t_{0}=100$ days) by the time the epidemic has already largely spread in the population, then social distancing measures less effective in reducing the final epidemic size. In both these cases, social distancing measures were not applied in an optimal manner, time wise. However, if social distancing is initiated at $t_{0}=80$ then the resulting epidemic is more effectively flattened. These observations are crystalized in Figure (2) (right), which suggests that there is in fact an optimal time, $t_{0}^{*}$, to introduce the social distancing so that the final epidemic size is minimized. Moreover, this figure suggests that the $t_{0}$ that minimizes the final epidemic size decreases as $R_{0}$ increases. That is, the more transmissible the disease is, the sooner 
social distancing measures should be initiated.
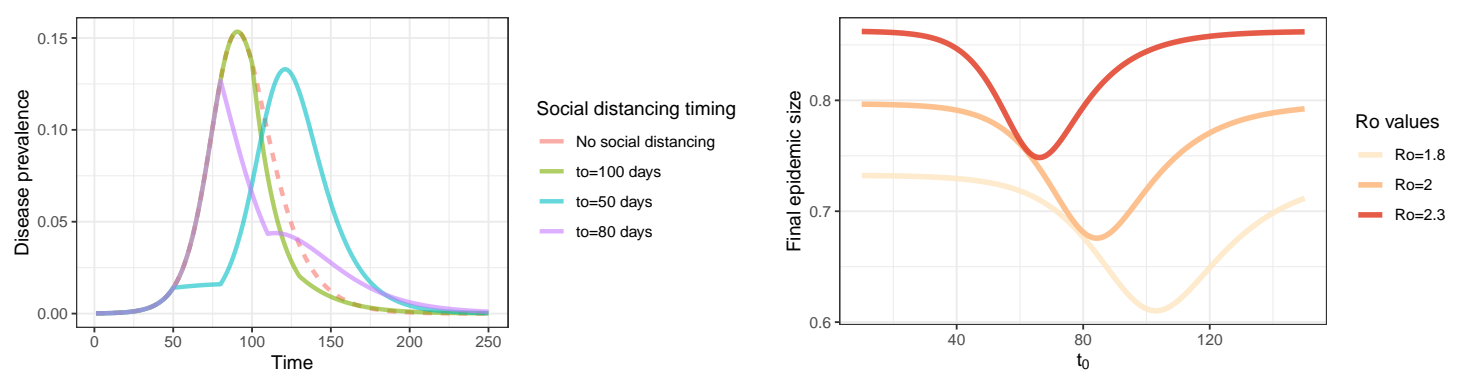

Figure 2: (left) Disease prevalence for different $t_{0}$ values and no social distancing (dashed line) with $R_{0}=2$. (right) Final epidemic size for different values of $t_{0}$ for three different values of $R_{0}$. For all simulations, $\gamma=1 / 10, T=30$ days, $r=0.55, I(0)=0.01 \%$.

\section{Simulations for the optimal $t_{0}$ : final size}

Building on the findings from the previous section, in what follows we conduct a more comprehensive analysis of how the optimal timing of social distancing depends on $R_{0}, r$ and $T$, when the objective is to minimize the final epidemic size. Figure (3) indicates that $R_{0}$ is the key factor in determining when to initiate social distancing, with $r$ and $T$ having virtually no effect. As $R_{0}$ increases, the sooner social distancing should start (see Figure (6)). Importantly, the optimal timing is never immediately after the beginning of the epidemic, unless $R_{0}$ is extremely large.

The effects of $r$ and $T$ are important in terms of the extent to which an optimally timed social distancing can reduce the final epidemic size (see Figure (8) in Appendix 3), with longer $T$ and smaller $r$ yielding greater reductions (up to $35 \%$ reductions in final size with the parameter ranges explored). Interestingly, the lower the $R_{0}$ the greater the reduction in final size. 

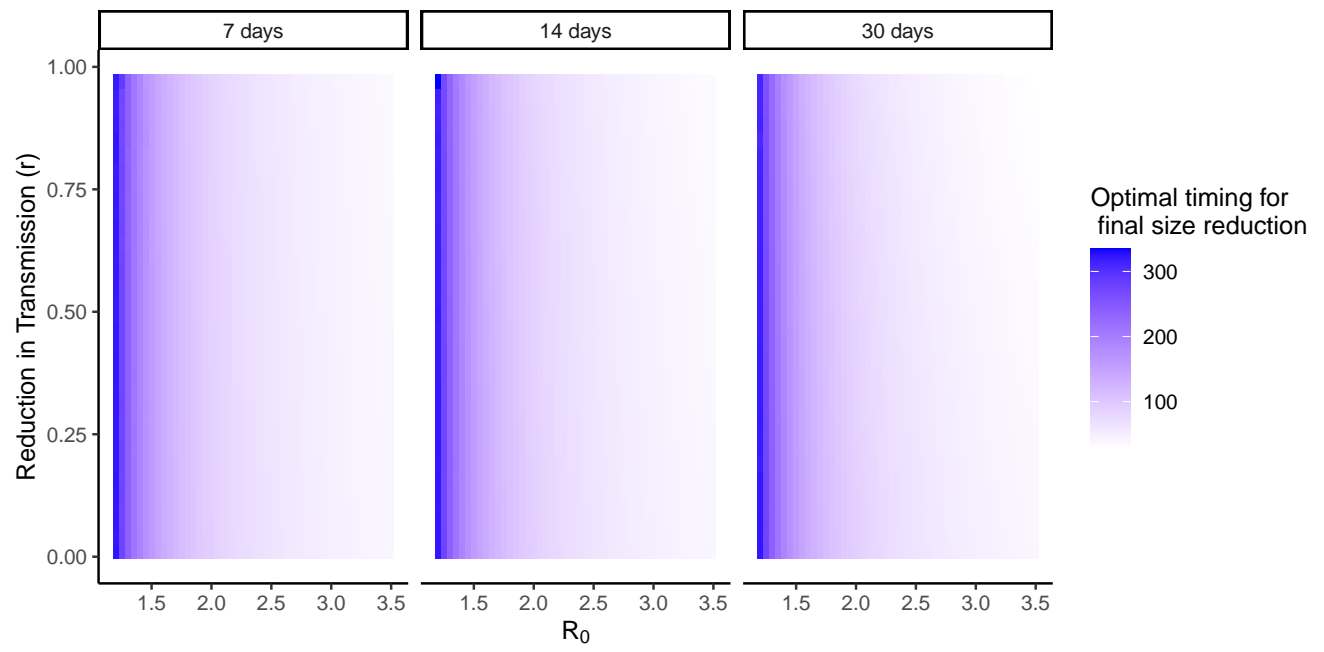

Figure 3: Optimal $t_{0}$ to initiate social distancing when the objective is to minimize the final epidemic size, as a function of $R_{0}$ (x axis), reduction in transmissibility during social distancing $r$ (y axis), and length of social distancing intervention (7, 14 and 30 days). As $R_{0}$ increases, the smaller the optimal time to initiate social distancing. For all simulations $\gamma=1 / 10$.

\section{Simulations for the optimal $t_{0}$ : flattening the epidemic}

\section{Optimal $t_{0}$ for peak delay}

Figure (4) also indicates that $R_{0}$ is the key factor in determining the optimal time to initiate social distancing if the goal is to delay the epidemic peak. Again, as $R_{0}$ increases, the sooner social distancing should start (see Figure (6)). Longer $T$ and smaller $r$ yield greater delays (up to 250 days), as expected (see Figure (9) in Appendix 3). Finally, the lower the $R_{0}$ the greater the delay in peak timing. 

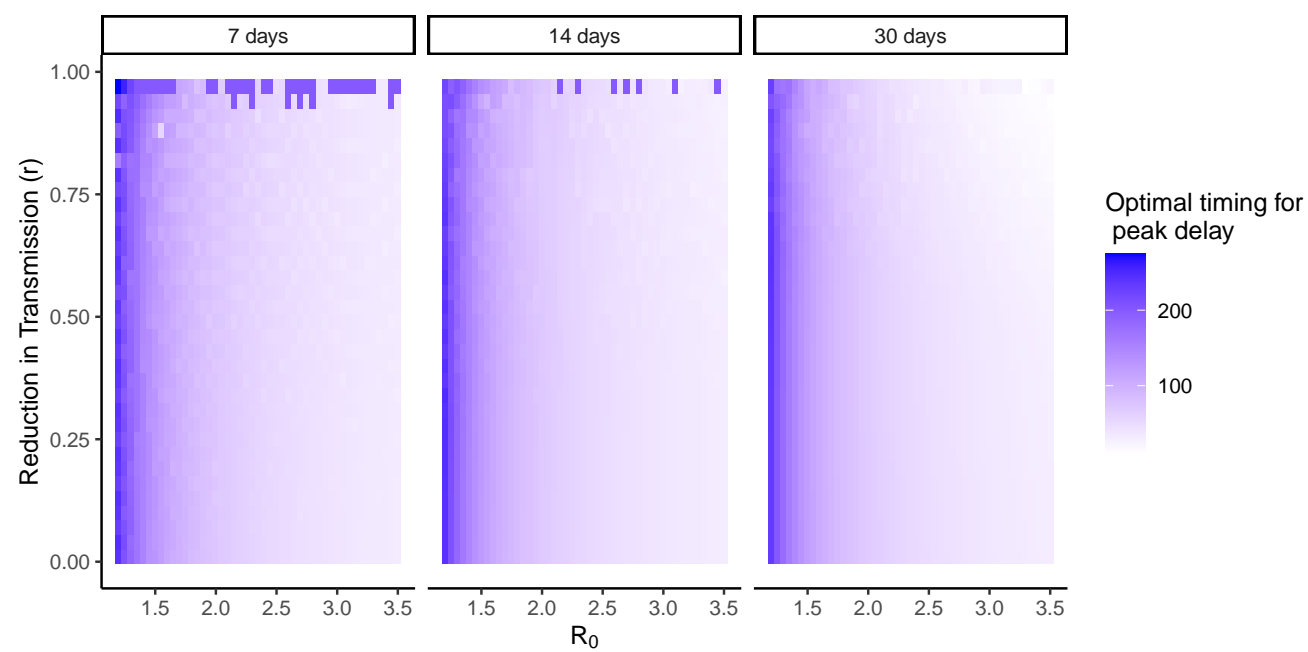

Figure 4: Optimal $t_{0}$ to initiate social distancing if the goal is to delay the epidemic peak, as a function of $R_{0}$ (x axis), reduction in transmissibility during social distancing $r$ (y axis), and length of social distancing intervention (7, 14 and 30 days). As $R_{0}$ increases, the smaller the optimal time to initiate social distancing. For all simulations $\gamma=1 / 10$.

\section{Optimal $t_{0}$ for peak reduction}

Figure (5) again indicates that $R_{0}$ is the main factor determining the optimal time to initiate social distancing if the goal is to reduce the epidemic peak. Once more, as $R_{0}$ increases, the sooner social distancing should start (see Figure (6)). Longer $T$ and smaller $r$ yield greater peak reductions (up to 60\% reductions), (see Figure (10) in Appendix 3). Finally, the higher the $R_{0}$ the greater the reduction in peak prevalence. 

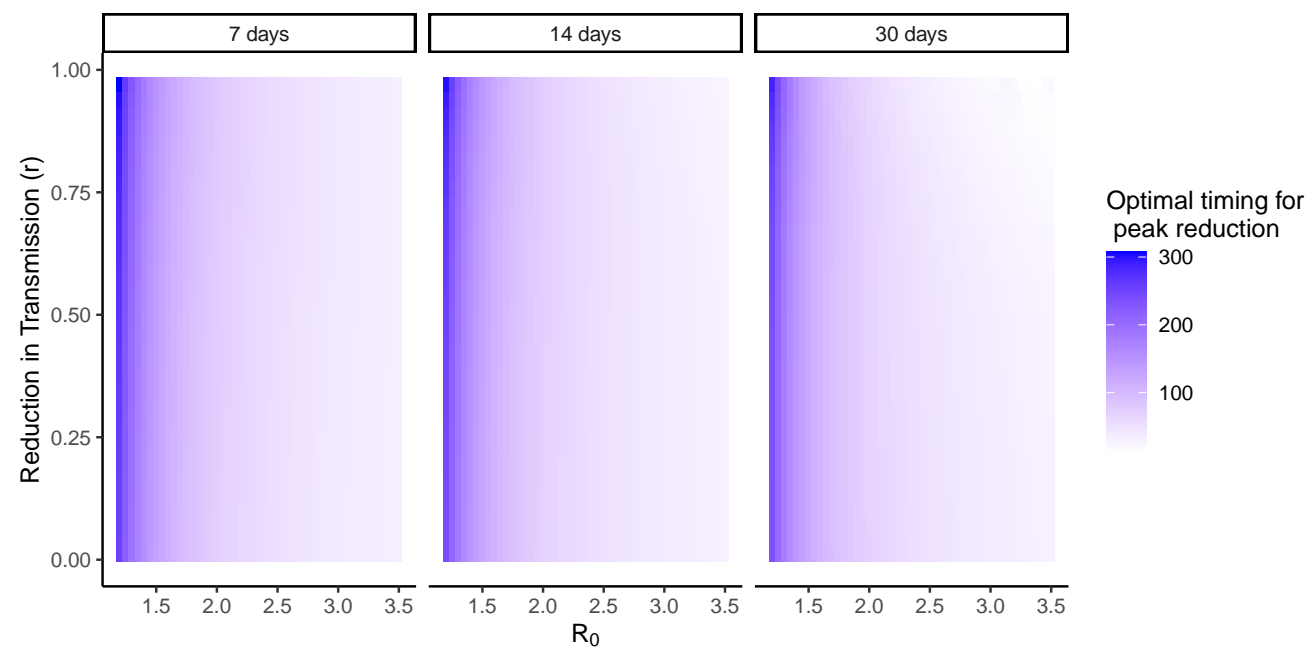

Figure 5: Optimal $t_{0}$ to initiate social distancing if the goal is to reduce the epidemic peak, as a function of $R_{0}$ (x axis), reduction in transmissibility during social distancing $r$ (y axis), and length of social distancing intervention (7,14 and 30 days). As $R_{0}$ increases, the smaller the optimal time to initiate social distancing. For all simulations $\gamma=1 / 10$.

Figure (6) shows that the optimal timing for social distancing decreases with $R_{0}$ in a quasi-exponential way, regardless of the epidemic containing strategy being employed. Interestingly, if the objective is to minimize final size, then the social distancing should start later compared to a social distancing strategy aimed at flattening the epidemic (either reducing or delaying the peak), with the differences decreasing as $R_{0}$ increases. For example, for an epidemic with $R_{0}=1.5$ (i.e., typical of a flu epidemic), the optimal social distance should be initiated 150 days after the start of the epidemic ${ }^{1}$ if the goal is to minimize the final epidemic size, whereas if the goal is to delay or reduce the peak as much as possible, social distancing should start at about 120 days.

\footnotetext{
${ }^{1}$ Strictly speaking, it is 150 days after $0.01 \%$ of the population has been infected, as $I(0)=0.01 \%$.
} 


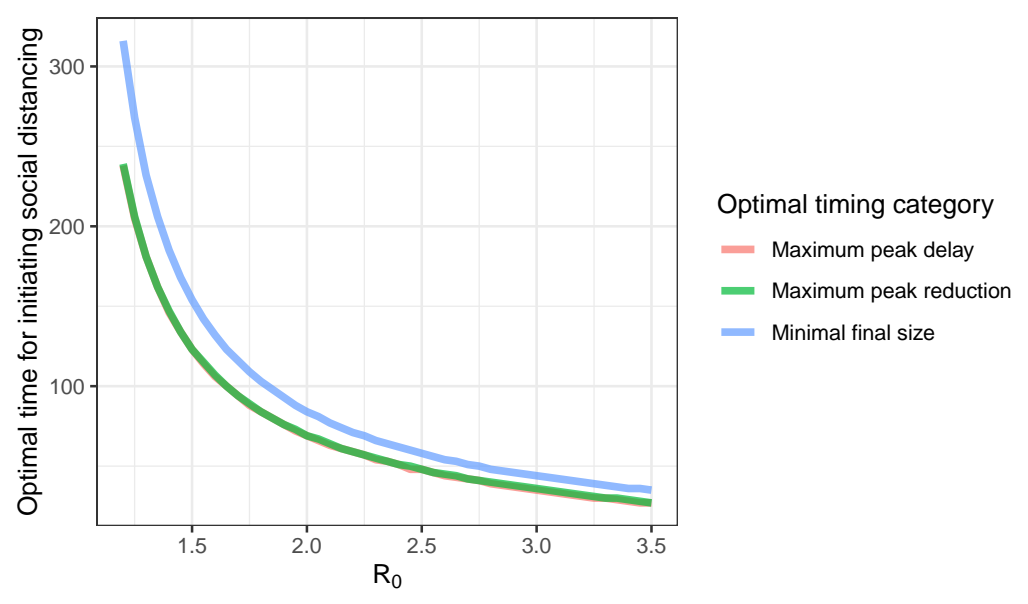

Figure 6: Optimal timing for social distancing vs $R_{0}$ for the three different types of optimal timing investigated, and corresponding to $T=30$ days and $r=0.55$ (very similar results are obtained for other values of $T$ and $r$ ). Note that the curves for "maximum peak delay" and "maximum peak reduction" are overlaid.

\section{Simulations for the optimal $t_{0}$ when an effective intervention is available in the future}

So far, the model has assumed that the only available intervention to curtail the spread of the disease is social distancing, and that other pharmacological interventions are not available at any point; or put differently, $t_{I}=\infty$. This assumption is most often wrong, as treatment options (e.g., vaccines, antivirals) typically become available at some point during the course of the epidemic. Here we explore a model with finite values of $t_{I}$, and see how the optimal social distancing time varies with $t_{I}$ and $R_{0}$. Figure (7) (left) shows that as $t_{I}$ decreases so does the optimal social distancing time if the goal is to reduce the final size (similar results are obtained if the goal is to flatten the epidemic curve, see Appendix 4). If the epidemic is only moderately transmissible (e.g., $1<R_{0}<2$ ) and pharmacological interventions are available relatively quickly (e.g., $t_{I}<200$ days), the optimal social distancing should be initiated almost immediately after the start of the epidemic. Intuitively, this result makes sense; if effective interventions become available soon after the start of the epidemic, a large second wave post social distancing is less likely because the intervention would prevent it.

Figure (7) (right) shows that the optimal timing for social distancing decreases sharply (particulalrly if the goal is to reduce the final size) as $t_{I}$ decreases, regardless of the epidemic containing strategy being employed. Note that if $t_{I}$ is large enough (i.e., the intervention comes long after the peak of the epidemic with no containing measures $[\sim$ 90 days, see Figure (2) (left)]), then the optimal time stops depending on the value of $t_{I}$. In the previous section we noted that if the objective is to minimize final size, then 
the social distancing should start later compared to a social distancing strategy aimed at flattening the epidemic. This is indeed the case for large $t_{I}$; however, for low values of $t_{I}$, the optimal time to minimize final size is shorter compared to a strategy aimed at flattening the epidemic.
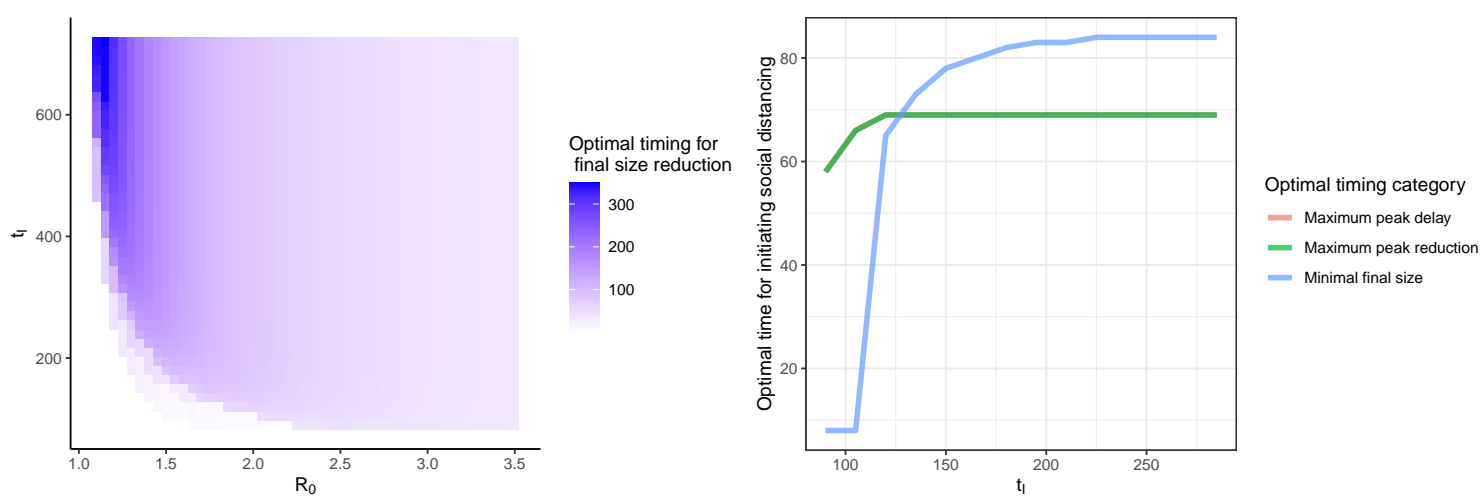

Figure 7: (left) Optimal $t_{0}$ to initiate social distancing to minimize final epidemic size as a function of $R_{0}$ (x axis), and time to pharmacological intervention $\left(t_{I}\right)$ (y axis). (right) Optimal timing for social distancing vs $t_{I}$ for the three different types of optimal timing investigated, and corresponding to $R_{0}=2$. With $\gamma=1 / 10, T=30, r=0.55, I(0)=$ $0.01 \%$. Note that the curves for "maximum peak delay" and "maximum peak reduction" are overlaid.

\section{Discussion}

We investigated the idea of optimally timing the start of temporary social distancing measures to more effectively hinder the spread of an epidemic. We find that, for a given disease transmissibility, and social distancing transmission-reduction effectiveness and length, there exists an optimal time to initiate the social distancing intervention. Regardless of the criteria used to define "optimum" timing, let it be final epidemic size reduction, epidemic peak delay or epidemic peak reduction, the timing is closely related to $R_{0}$, and not so much with $T$ and $r$. Moreover, we find that the optimal time follows an approximate exponential decay relationship with $R_{0}$, such that the higher the $R_{0}$ the sooner social distancing measures should be initiated.

The factors $r$ and $T$ determine the extent to which optimally timed social distancing reduces the final epidemic size or flattens the epidemic curve. Larger reductions in transmissibility and social distancing of longer durations lead, as expected, to grater reductions in final size and more flattening of the epidemic. Interestingly, the lower the $R_{0}$ the greater the potential reductions of optimally timed social distancing measures in final size and delay in peak prevalence, whereas the higher the $R_{0}$ the greater the reduction in peak prevalence. 
If pharmacological interventions, such as effective treatments and/or vaccines, become available at scale, the time point at which they do so has also important implications for the optimal time for social distancing. The sooner pharmacological interventions become available, the sooner social distancing should be introduced, particularly if the pharmacological interventions can be deployed around or right after the time the epidemic would peak in the absence of any sort of containing intervention.

It is important to remark that these results stem from very idealized assumptions about the course of the epidemic. The inclusion of transmission or contact level heterogeneities within the population [19] (e.g., age structure) may play a significant role in the quantitative description of the results in this paper, as would the inclusion of seasonality effects. Additionally, since we are using a deterministic mean-field approach, these analyses do not account for the effects of stochastic fluctuations (which could lead to epidemic extinction if the number of infected is low enough during the social distancing phase) and as a consequence, the findings herein apply more closely to very large populations (i.e., in the thermodynamic limit) where stochastic fluctuations are less relevant. Similarly, the model assumes homogeneous mixing among individuals, thus the contact network structure of the population is not accounted for; as a result this model cannot realistically predict the epidemic-hampering effects that social distancing measures (e.g., limiting the size of crowds) have in the context of contact structured populations [20].

An important assumption in this exercise of finding an optimal time for initiating social distancing, is that individuals that are infected or immune are all perfectly ascertained and tracked (i.e., no underreporting of cases) such that decision makers have perfect knowledge of the $R_{0}$ and the effective reproduction number (via estimation from epidemic data), as well as the number of new cases at any given point. Early in an epidemic, particularly when in the presence of a novel pathogen like SARS-CoV-2, there is typically a large amount of uncertainty around most epidemiological parameters, especially $R_{0}$ $[9,23]$. This uncertainty is the result of several factors, including lack of reliable data on the true number of cases, differences in the formulations and assumptions underlying the statistical models used to estimate $R_{0}$, and the geographic heterogeneity of the disease spreading patterns. This uncertainty around the value of $R_{0}$ should also be carried over to the estimate of the optimal time for initiating social distancing. Hence, assuming the true $R_{0}$ lies within a range (e.g., $95 \%$ confidence intervals), the estimation of the optimal $t_{0}$ should account for this uncertainty. Moreover, if a (Bayesian posterior) distribution is available for $R_{0}$, then one could also get a distribution for the optimal $t_{0}$, which would in turn allow to compute the probability that the optimal $t_{0}$ is within a given range, or lower/higher than a given time of interest (e.g., two weeks from now). Then, it would be up to the decision makers to determine what level of uncertainty is considered acceptable in order to proceed with a given social distancing strategy. Of note, if the level of uncertainty on $R_{0}$ is very large and a decision needs to be made promptly, it is arguably better to initiate social distancing sooner, as opposed to later, than the optimal $t_{0}$ inferred from the available data; therefore, a conservative approach could be to assume $R_{0}$ is equal to the upper bound of its $\% 95$ confidence interval, and compute the corresponding (shorter) 
optimal $t_{0}$.

The findings herein serve to show the important "proof of concept" of an optimal time to initiate social distancing, which arises as an interesting and potentially useful feature of a simple epidemiological model. Our hope is that these insights inspire other researchers to investigate the existence of optimal (or just more effective) times to implement social distancing measures within the context of more complex and data-driven epidemiological models, as to better serve decision makers developing strategic policies to mitigate the extent of current and future epidemics.

\section{Acknowledgments}

We would like to thank Andres Gomez-Lievano for helpful comments and suggestions on a previous draft of this manuscript.

\section{References}

[1] Covid-19 prevention and treatment what you need to know about prevention and treatment of covid-19 in massachusetts. Mass.gov.

[2] Interim us guidance for risk assessment and public health management of persons with potential coronavirus disease 2019 (covid-19) exposures: Geographic risk and contacts of laboratoryconfirmed cases. Mass.gov.

[3] R.M. Anderson and R.M. May. Infectious Diseases of Humans: Dynamics and Control (Part 1, Chapters 4-7). Oxford Science Publications. Oxford University Press, 1992.

[4] Roy M Anderson, Hans Heesterbeek, Don Klinkenberg, and T Déirdre Hollingsworth. How will country-based mitigation measures influence the course of the covid-19 epidemic? The Lancet, 395(10228):931-934, 2020.

[5] Simiao Chen, Juntao Yang, Weizhong Yang, Chen Wang, and Till Bärnighausen. Covid-19 control in china during mass population movements at new year. The Lancet, 2020.

[6] Gerardo Chowell, Luis MA Bettencourt, Niall Johnson, Wladimir J Alonso, and Cecile Viboud. The 1918-1919 influenza pandemic in england and wales: spatial patterns in transmissibility and mortality impact. Proceedings of the Royal Society B: Biological Sciences, 275(1634):501-509, 2008.

[7] Neil M Ferguson, Daniel Laydon, Gemma Nedjati-Gilani, Natsuko Imai, Kylie Ainslie, Marc Baguelin, Sangeeta Bhatia, Adhiratha Boonyasiri, Zulma Cucunubá, Gina CuomoDannenburg, et al. Impact of non-pharmaceutical interventions (npis) to reduce covid19 mortality and healthcare demand. Imperial College, London. DOI: https://doi. org/10.25561/77482, 2020.

[8] Centers for Disease Control, Prevention (CDC, et al. Update: severe acute respiratory syndrome-toronto, canada, 2003. MMWR. Morbidity and mortality weekly report, 52(23):547, 2003. 
[9] Laurent Hébert-Dufresne, Benjamin M Althouse, Samuel V Scarpino, and Antoine Allard. Beyond $r_{-} 0$ : the importance of contact tracing when predicting epidemics. arXiv preprint arXiv:2002.04004, 2020.

[10] Marco Arieli Herrera-Valdez, Maytee Cruz-Aponte, and Carlos Castillo-Chavez. Multiple outbreaks for the same pandemic: Local transportation and social distancing explain the different" waves" of a-h1n1pdm cases observed in méxico during 2009. Mathematical Biosciences E Engineering, 8(1):21-48, 2011.

[11] Stephen M Kissler, Christine Tedijanto, Edward Goldstein, Yonatan H Grad, and Marc Lipsitch. Projecting the transmission dynamics of sars-cov-2 through the post-pandemic period. medRxiv, 2020.

[12] Stephen M Kissler, Christine Tedijanto, Marc Lipsitch, and Yonatan Grad. Social distancing strategies for curbing the covid-19 epidemic. medRxiv, 2020.

[13] Savi Maharaj and Adam Kleczkowski. Controlling epidemic spread by social distancing: Do it well or not at all. BMC Public Health, 12(1):679, 2012.

[14] Apoorva Mandavilli. Wandering about social distancing. The New York Times.

[15] David MartÂn-Calvo, Alberto Aleta, Alex Pentland, Yamir Moreno, and Esteban Moro. Effectiveness of social distancing strategies for protecting a community from a pandemic with a datadriven contact network based on census and real-world mobility data. 2020 .

[16] Corey M Peak, Rebecca Kahn, Yonatan H Grad, Lauren M Childs, Ruoran Li, Marc Lipsitch, and Caroline O Buckee. Modeling the comparative impact of individual quarantine vs. active monitoring of contacts for the mitigation of covid-19. medRxiv, 2020.

[17] R Core Team. R: A Language and Environment for Statistical Computing. R Foundation for Statistical Computing, Vienna, Austria, 2020.

[18] Denise Roland and Drew Hinshaw. Social distancing is new coronavirus buzz phrase. does it work? The Wall Street Journal.

[19] Eunha Shim. Optimal strategies of social distancing and vaccination against seasonal influenza. Mathematical Biosciences \& Engineering, 10(5\&6):1615-1634, 2013.

[20] Guillaume St-Onge, Vincent Thibeault, Antoine Allard, Louis J Dubé, and Laurent HébertDufresne. School closures, event cancellations, and the mesoscopic localization of epidemics in networks with higher-order structure. arXiv preprint arXiv:2003.05924, 2020.

[21] Stuart A. Thompson and Yaryna Serkez. Deciding how much distance you should keep. The New York Times.

[22] Stuart A. Thompson and Yaryna Serkez. How has your state reacted to social distancing? The New York Times.

[23] Jacco Wallinga and Marc Lipsitch. How generation intervals shape the relationship between growth rates and reproductive numbers. Proceedings of the Royal Society B: Biological Sciences, 274(1609):599-604, 2007. 
[24] Jacco Wallinga, Michiel van Boven, and Marc Lipsitch. Optimizing infectious disease interventions during an emerging epidemic. Proceedings of the National Academy of Sciences, 107(2):923-928, 2010.

[25] Juanjuan Zhang, Maria Litvinova, Yuxia Liang, Yan Wang, Wei Wang, Shanlu Zhao, Qianhui $\mathrm{Wu}$, Stefano Merler, Cecile Viboud, Alessandro Vespignani, et al. Age profile of susceptibility, mixing, and social distancing shape the dynamics of the novel coronavirus disease 2019 outbreak in china. medRxiv, 2020.

[26] Yuzhen Zhang, Bin Jiang, Jiamin Yuan, and Yanyun Tao. The impact of social distancing and epicenter lockdown on the covid-19 epidemic in mainland china: A data-driven seiqr model study. medRxiv, 2020. 


\section{Appendix 1: Derivation of final size expression}

Here we find an analytical expression for the final epidemic size (i.e., the fraction of infected individuals at the end of the epidemic) in the context of temporal social distancing measure. For this derivation, we assume that $t_{I}=\infty$. In principle, this expression should hold a connection between the final epidemic size and $t_{0}$. The ODE system in (2)-(3) has a basic reproductive number given by $R_{0}=\beta(t) / \gamma$ (with $t \approx 0$ ). With no social distancing being implemented, that is $\beta(t)=\beta$, the final size relation for the system (2)-(3) is:

$$
e^{-y R_{0}}=1-y
$$

Lets now compute the same final size relation for when the transmission rate corresponds to the piecewise function shown above in Figure(1), i.e., it is reduced for a period of time $T$. Assume that at the beginning of the epidemic the population was divided into susceptible $\left(S_{0}\right)$ and infected $\left(I_{0}\right)$, such that $N=I_{0}+S_{0}$. We proceed to compute the final size as follows:

$$
\begin{aligned}
\frac{d I}{d S} & =-1+\frac{\gamma}{\beta(t) S} \\
\Longrightarrow d I & =\left(-1+\frac{\gamma}{\beta(t) S}\right) d S
\end{aligned}
$$

Integrating with respect to time until time $\tau$, taking $\tau>t_{0}+T$, we get

$$
\begin{aligned}
I(\tau)-I_{0}= & S_{0}-S(\tau)+\gamma\left(\frac{1}{\beta} \int_{0}^{t_{0}} \frac{d S}{S}+\frac{1}{\beta_{r}} \int_{t_{0}}^{t_{0}+T} \frac{d S}{S}+\frac{1}{\beta} \int_{t_{0}+T}^{\tau} \frac{d S}{S}\right) \\
I(\tau)-N= & -S(\tau)+\frac{\gamma}{\beta}\left[\ln \left(S\left(t_{0}\right)\right)-\ln (S(0))\right]+\frac{\gamma}{\beta_{r}}\left[\ln \left(S\left(t_{0}+T\right)\right)-\ln \left(S\left(t_{0}\right)\right)\right]+ \\
& \frac{\gamma}{\beta}\left[\ln (S(\tau))-\ln \left(S\left(t_{0}+T\right)\right)\right]
\end{aligned}
$$

Assuming that $S(0) \approx 1$ and taking the limit $\tau \rightarrow \infty$, and after a number of algebraic manipulations, while defining $f$ as the final size proportion of infected (attack rate) with SD, we obtain

$$
e^{-R_{0} f}=(1-f)\left(\frac{S\left(t_{0}\right)}{S\left(t_{0}+T\right)}\right)^{\left(1-\frac{1}{r}\right)}
$$

where $f$ is the epidemic final size (the proportion of individuals that got infected during the outbreak). Thus, when the transmission rate corresponds to the piecewise function shown in Figure (1), the final size expression is instead given by:

$$
e^{-R_{0} f}=(1-f) \sigma^{\omega} .
$$

where

$$
\sigma=\frac{S\left(t_{0}\right)}{S\left(t_{0}+T\right)} \quad \text { and } \quad \omega=1-1 / r .
$$

To check the soundness of this expression, note that if $r=1$ (social distancing was completely ineffective in reducing transmission) then $\omega=0$, and we obtain the classical expression for the final size in Equation (5). Another similar instance is given by $\sigma=1$ which corresponds to $T=0$.

Equation (7) implicitly relates $f$ and $t_{0}$. The functional form of $f\left(t_{0}\right)$ would serve to derive a condition on $t_{0}$ that minimizes $f$, that is, find $t_{0}^{*}$ such that $\min \left[f\left(t_{0}\right)\right]=f\left(t_{0}^{*}\right)$. However, such transcendental equation does not allow us to isolate $f$. 


\section{Appendix 2: Numerical simulations setup}

For each value of $t_{0} \in(0-350), R_{0} \in(1.2-3.5), r \in(0.01-0.99)$ and $T \in\{7,14$ and 30 days $\}$, the model in system in (2)-(3) was simulated in R [17] for 1825 days (5 years). For all simulations $S(0)=.9999, I(0)=0.001$, and $\gamma=1 / 10$.

For each combination of $R_{0}, T$ and $r$, the value of $t_{0}$ corresponding to 1) the minimal final size at 5 years $\left.\left(t_{01}^{*}\right), 2\right)$ the maximum delay in incidence peak time $\left(t_{02}^{*}\right)$, and 3$)$ the minimum incidence peak $\left(t_{03}^{*}\right)$ was obtained. To quantify the effect of these three versions of optimal social distancing, a base model with no social distancing was also simulated for each combination of $R_{0}, T$ and $r$.

The final size reduction assuming optimal SD timing was computed as:

$$
\left.1-\left(\text { final.size }\left(t_{01}^{*}\right)\right) / \text { final.size }(T=0)\right) \text {. }
$$

The incidence peak delay assuming optimal SD timing was computed as:

$$
\text { peak.time }\left(t_{02}^{*}\right) \text { - peak.time }(T=0) \text {. }
$$

The incidence peak reduction assuming optimal SD timing was computed as:

$$
\left.1-\left(\operatorname{peak}\left(t_{03}^{*}\right)\right) / \operatorname{peak}(T=0)\right) .
$$

\section{Appendix 3: Effect of optimal $t_{0}$ in reducing final epidemic size and flattening the epidemic}

Figure (8) suggests, not surprisingly, that the effects of $r$ and $T$ are important in terms of the extent to which an optimally timed social distancing can reduce the final epidemic size, with longer $T$ and smaller $r$ yielding greater reductions (up to $35 \%$ reductions in final size with the parameter ranges explored). Interestingly, the lower the $R_{0}$ the greater the reduction in final size. 

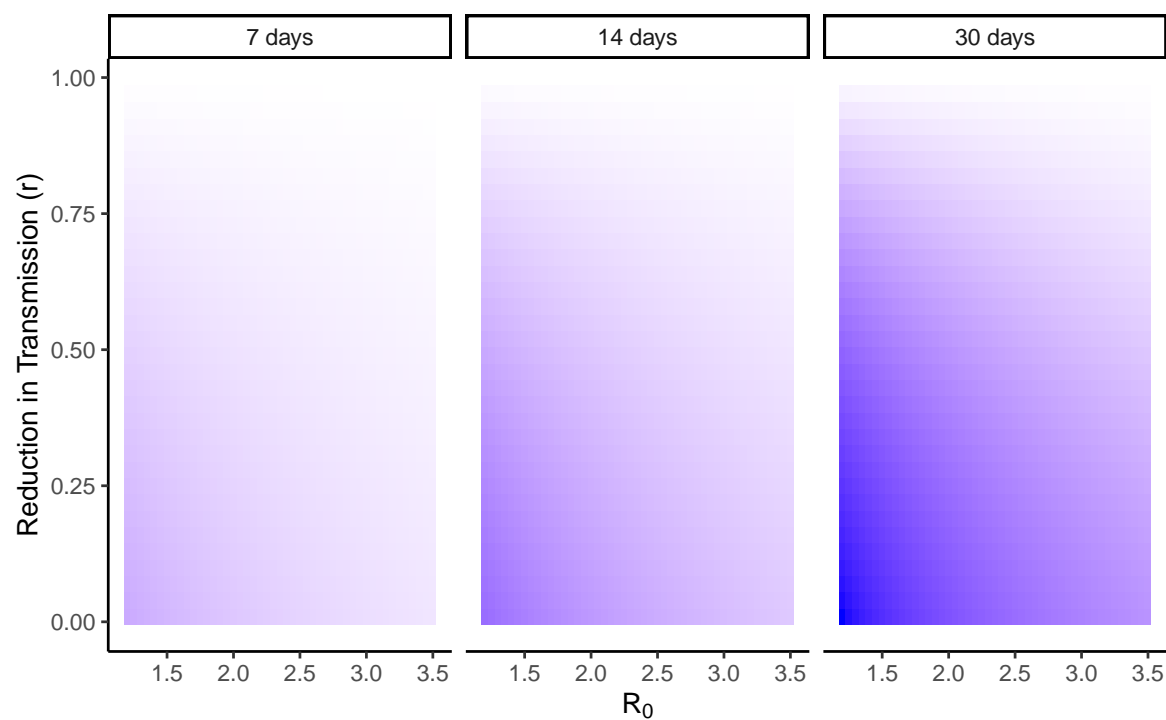

Final size reduction

0.3

0.2

0.1

Figure 8: Reduction in final size corresponding to optimal $t_{0}$ to initiate social distancing as a function of $R_{0}$ (x axis), reduction in transmissibility during social distancing $r$ (y axis), and length of social distancing intervention (7,14 and 30 days). For all simulations $\gamma=1 / 10$.

Figure (9) indicates that longer $T$ and smaller $r$ yield greater delays (up to 250 days), as expected. Finally, the lower the $R_{0}$ the greater the delay in peak timing. 

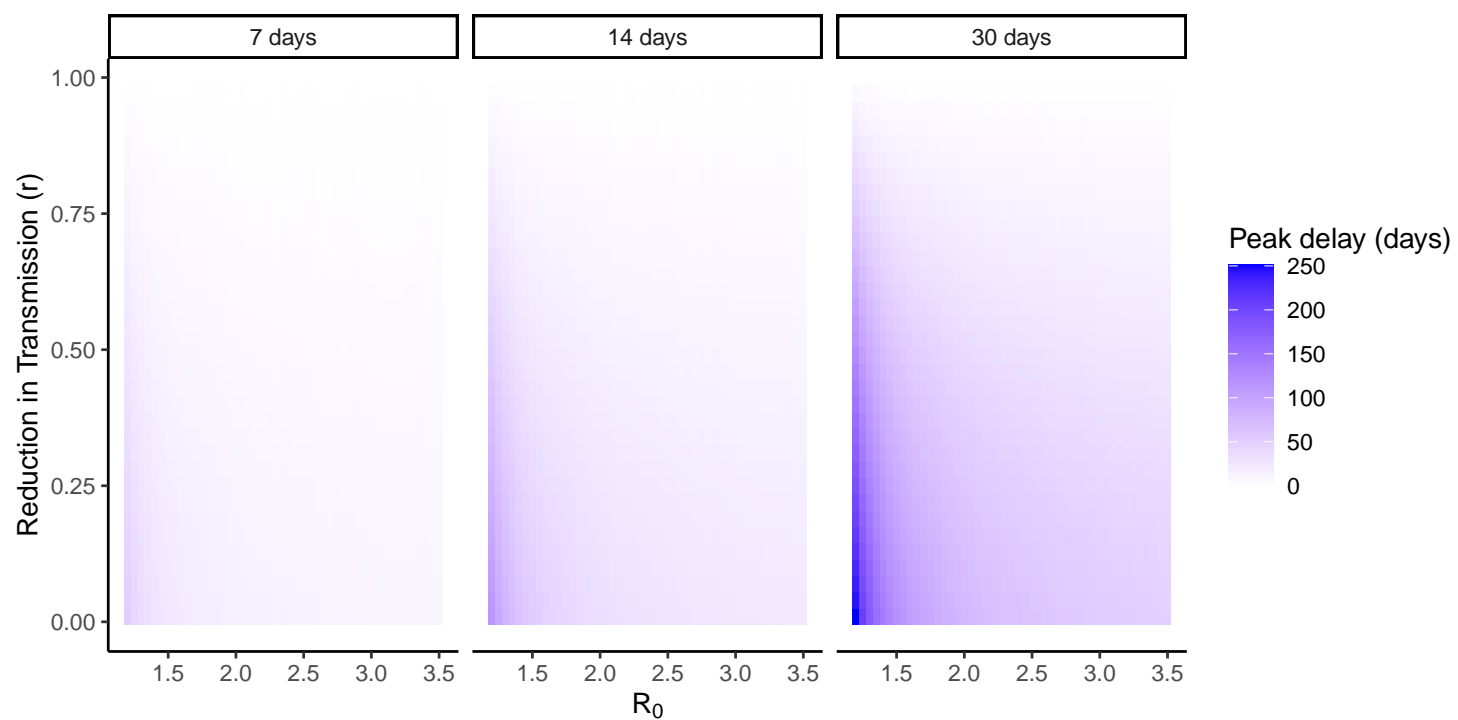

Figure 9: Delay in peak time corresponding to optimal $t_{0}$ to initiate social distancing as a function of $R_{0}$ (x axis), reduction in transmissibility during social distancing $r$ (y axis), and length of social distancing intervention (7, 14 and 30 days). For all simulations $\gamma=1 / 10$.

Figure (10) indicates that longer $T$ and smaller $r$ yield greater peak reductions (up to $60 \%$ reductions), as expected. Finally, the higher the $R_{0}$ the greater the reduction in peak prevalence. 

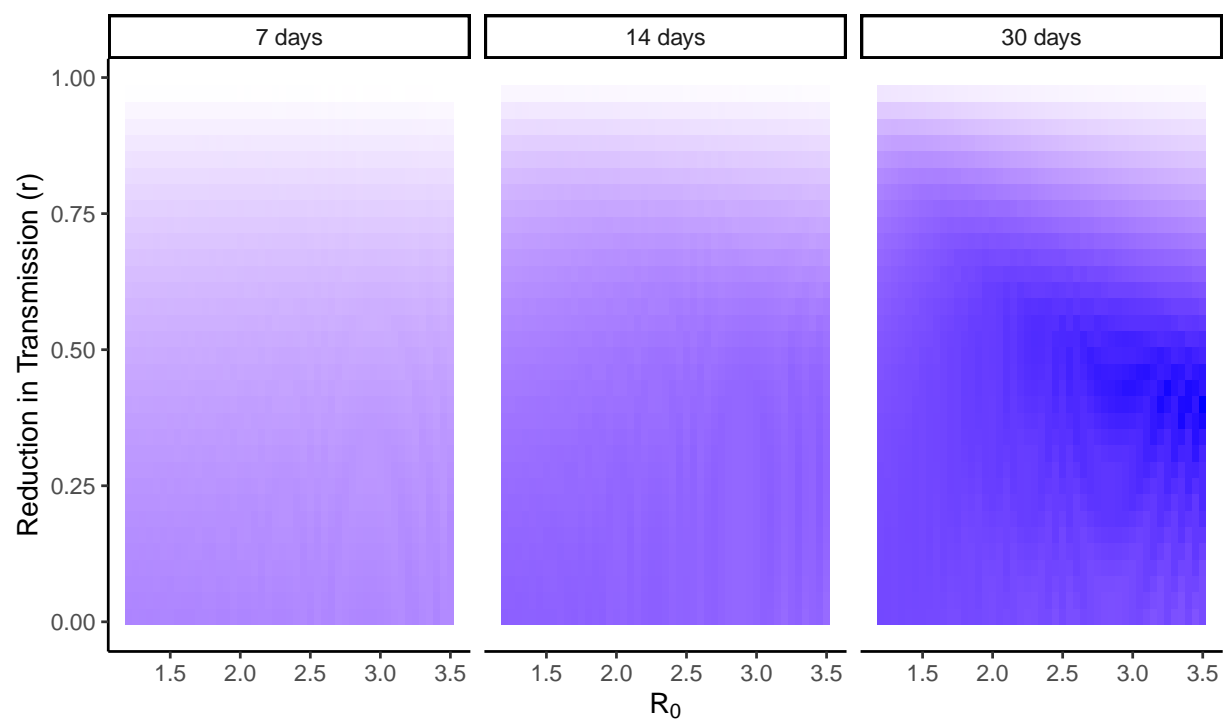

Peak reduction

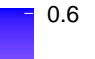

0.4

0.2

Figure 10: Reduction in peak time corresponding to optimal $t_{0}$ to initiate social distancing as a function of $R_{0}$ (x axis), reduction in transmissibility during social distancing $r$ (y axis), and length of social distancing intervention (7, 14 and 30 days). For all simulations $\gamma=1 / 10$.

\section{Appendix 4: Simulations for the optimal $t_{0}$ to flatten the epidemic when an effective intervention is available in the feature}

Figure (11) shows that as $t_{I}$ decreases so does the optimal social distancing time if the goal is to flatten the epidemic curve. 

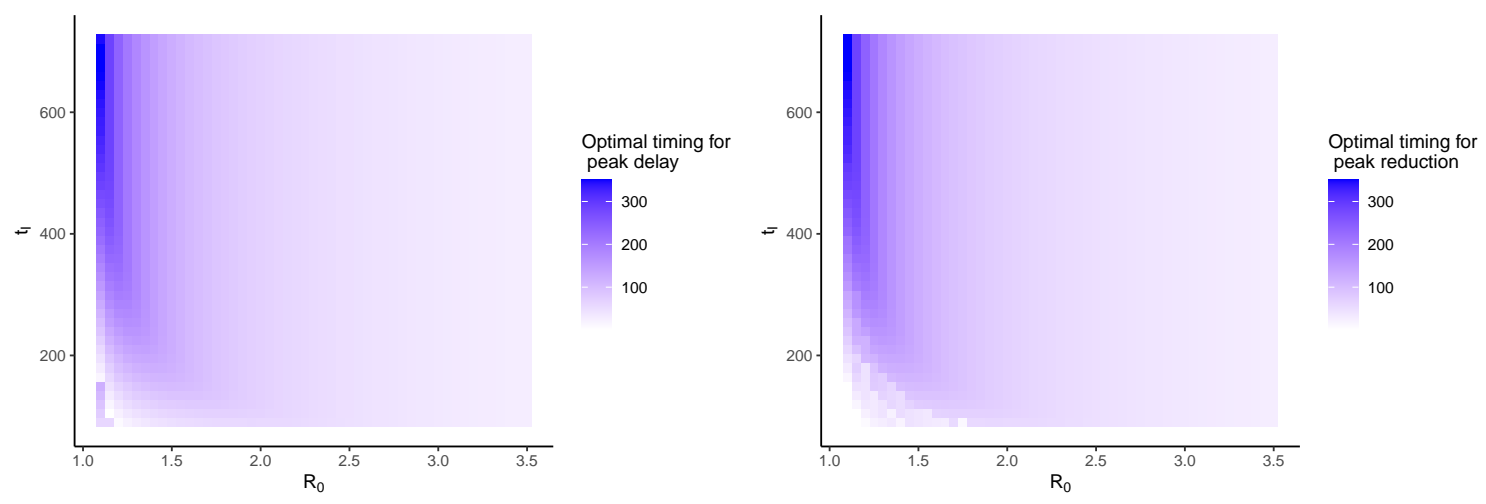

Figure 11: (left) Optimal $t_{0}$ to initiate social distancing to maximize the delay of the epidemic peak as a function of $R_{0}$ (x axis), and time to pharmacological intervention $\left(t_{I}\right)$ (y axis). (right) Optimal $t_{0}$ to initiate social distancing to maximize the reduction of the epidemic peak as a function of $R_{0}$ (x axis), and time to pharmacological intervention $\left(t_{I}\right)$ (y axis). With $\gamma=1 / 10, T=30, r=0.55, I(0)=0.01 \%$. 